\title{
L'EXAMEN DES ACTES ÉTATIQUES AU REGARD DE LA CHARTE DES DROITS FONDAMENTAUX DE L'UNION EUROPÉENNE
}

\author{
FABRICE PICOD ${ }^{1}$ \\ L'Université Paris 2 Panthéon-Assas \\ Fabrice.Picod@u-paris2.fr
}

\section{Résumé}

La Charte des droits fondamentaux s'applique sans difficulté aux actes des institutions, organes et agences de l'Union. Mais son champ d'application s'étend également aux actes adoptés par les États membres lorsqu'ils mettent en œuvre le droit de l'Union. La Cour de justice a été obligée de préciser, non sans difficultés, quels types d'actes étatiques impliquent l'application du droit de l'Union et sont donc soumis aux exigences de la Charte.

\section{Mots clés}

Charte des droits fondamentaux de l'Union européenne; actes étatiques; actes étatique qui appliquent le droit dérivé; jurisprudence Akerberg Fransson; actes étatiques qui développent d'autres types de normes de l'UE.

1 Professeur à l'Université Paris 2 Panthéon-Assas. Chaire Jean Monnet. Directeur du Centre de droit européen. 


\section{EL EXAMEN DE LOS ACTOS ESTATALES A LA LUZ DE LA CARTA DE DERECHOS FUNDAMENTALES DE LA UNIÓN EUROPEA}

\section{Resumen}

La Carta de Derechos Fundamentales se aplica sin dificultad a los actos de las instituciones órganos y organismos de la Unión. Pero su ámbito de aplicación también se extiende a los actos adoptados por los Estados miembros cuando aplican derecho de la Unión. El Tribunal de Justicia se ha visto obligado a clarificar en su jurisprudencia, no sin dificultades, qué tipos de actuaciones estatales suponen una aplicación del derecho de la Unión y quedan, por tanto, sometidos a las exigencias de la Carta.

\section{Palabras clave}

Carta de Derechos Fundamentales de la Unión Europea; actos estatales; actos estatales que aplican derecho derivado; jurisprudencia Akerberg Fransson; actos estatales que desarrollan otro tipo de normas de la UE.

\section{THE EXAMINATION OF STATE ACTS WITH REGARD TO THE CHARTER OF FUNDAMENTAL RIGHTS OF THE EUROPEAN UNION}

\section{Abstract}

The Charter of Fundamental Rights applies without difficulty to the acts of the institutions, organs and agencies of the Union. However, its scope also extends to acts adopted by Member States when they apply Union law. The Court of Justice has been obliged to clarify in its jurisprudence, not without difficulties, what types of State actions involve the application of Union law and are, therefore, subject to the requirements of the Charter.

\section{Keywords}

Charter of Fundamental Rights of the European Union; State acts; State acts that apply secondary law; jurisprudence Akerberg Fransson; State acts that develop other types of EU rules. 


\section{SOMMAIRE}

I. CADRE GÉNÉRAL ET CRITÈRES D'EXAMEN DES ACTES ÉTATIQUES MIS EN CAUSE. II. EXAMEN DES ACTES ÉTATIQUES DE MISE EN OEUVRE DU DROIT DÉRIVÉ DE L'UNION EUROPÉENNE. III. EXAMEN DES ACTES ÉTATIQUES DE MISE EN OEUVRE DES AUTRES SOURCES DE DROIT DE L'UNION EUROPÉENNE. BIBLIOGRAPHIE.

La Charte des droits fondamentaux de l'Union européenne n'a pas été conçue comme un texte qui devait contraindre à titre principal les Etats membres de l'Union européenne dans la mesure où ces derniers devaient déjà respecter les droits reconnus dans la Convention européenne des droits de l'homme auxquels ils sont tous parties et les droits qui sont garantis par leurs propres constitutions nationales.

Ce qui a été recherché dans ce texte est avant tout une garantie de la protection des droits fondamentaux que les institutions de l'Union européenne devraient, dans l'élaboration du droit dérivé, impérativement respecter. L'adoption d'une Charte des droits fondamentaux de l'Union européenne, au-delà de son caractère symbolique, devait constituer un progrès dans le système constitutionnel de l'Union ${ }^{2}$. La Charte devait ainsi combler une lacune tenant au fait que l'action des institutions, organes et organismes de l'Union n'était qu'imparfaitement encadrée au regard des droits fondamentaux.

En même temps, il aurait été regrettable qu'un texte de progrès d'une telle envergure ne contraigne pas également les Etats membres de l'Union européenne compte tenu de l'imbrication de leurs missions avec celles des institutions de l'Union dans la réalisation des objectifs assignés à l'Union européenne par les traités constitutifs.

C'est l'art. 51 de la Charte des droits fondamentaux de l'Union européenne, relatif à son champ d'application, qui désigne les personnes qui sont tenues au respect de ses dispositions ${ }^{3}$. Cet art. vise expressément deux catégories de sujets d'obligations bien distinctes: les institutions, organes et organismes de l'Union, d'une part, et les Etats membres, d'autre part. Tandis que les premiers sont soumis au respect de la Charte dans le respect du

\footnotetext{
2 Voir notamment Rideau (2009).

3 Voir notamment Borowsky (2014), Lenaerts (2017), Ovadek (2017), Picod (2019), Ward (2014).
} 
principe de subsidiarité, les seconds y sont soumis «lorsqu'ils mettent en œuvre le droit de l'Union» ${ }^{4}$.

La contrainte qu'a fait peser l'art. 51 de la Charte des droits fondamentaux sur les Etats membres ne pouvait être qualifiée d'inédite dans la mesure où elle se situait dans le prolongement de la jurisprudence de la Cour de justice relative à la protection des droits fondamentaux au moyen des principes généraux du droit qui avait déjà donné lieu à des controverses doctrinales. De vives critiques avaient en effet été formulées par deux auteurs à propos de la finalité de l'extension à l'activité des États membres de la jurisprudence de la Cour de justice relative aux droits fondamentaux (Coppel et O'Neill, 1992: 669 et s.) mais elles avaient donné lieu à une analyse minutieuse et globalement convaincante réfutant point par point les critiques formulées par les premiers auteurs 5 .

Dès 1975, dans son arrêt Rutili, la Cour de justice avait précisé, au sujet de la mise en œuvre de la Directive 64/221 sur la coordination des mesures relatives à l'ordre public prises à l'encontre des étrangers, que les atteintes portées aux droits de l'homme, pour des besoins d'ordre public, ne pouvaient dépasser, conformément aux exigences de la Convention européenne des droits de l'homme, ce qui était nécessaire à la sauvegarde des besoins ${ }^{6}$.

C'est surtout lorsque les Etats membres eurent à prendre des mesures d'application de la réglementation de l'Union européenne qu'ils se sont vu imposer, au même titre que les institutions de l'Union auteurs de celle-ci, de respecter les droits fondamentaux reconnus au moyen de principes généraux de droit ${ }^{7}$. En effet, lorsqu'un Etat membre adoptait des mesures d'application d'une réglementation de droit de l'Union européenne, il était logique de le soumettre à un régime comparable à celui imposé à l'institution de l'Union européenne auteur de l'acte de référence en ce qui concerne le respect des principes généraux de droit de l'Union européenne et, plus particulièrement, des droits fondamentaux reconnus sur ce fondement. En ce sens, la Cour de justice avait considéré, avant l'adoption de la Charte, que les exigences découlant de la protection des droits fondamentaux dans l'ordre juridique communautaire «lient également les Etats membres lorsqu'ils mettent en

4 Voir notamment Besselink (2001), Picod (2018), Safjan, Düsterhaus, Guerin (2016), Tizzano (2014).

5 Voir Weiler et Lockhart, N. J. S. (1995).

6 Arrêt du 28 octobre 1975, Rutili, 36/75, EU:C:1975:137, att. 32.

7 Arrêt du 13 juillet 1989, Wachauf, 5/88, EU:C:1989:321, point 19. Voir également arrêts du 24 mars 1994, Bostock, C-2/92, EU:C:1994:116, point 16; et du 17 avril 1997, EARL de Kerlast, C-15/95, EU:C:1997:196, point 36. 
œuvre des réglementations communautaires ${ }^{8}$. L'exigence de respect des droits fondamentaux s'est également logiquement imposée lorsque les Etats membres transposaient une directive dans leur droit national', la transposition pouvant être considérée comme une opération de mise en œuvre de la réglementation de l'Union européenne.

C'est lorsque les Etats membres n'agissaient pas en application d'une réglementation de l'Union européenne que la Cour de justice avait pris position en choisissant une formule nécessairement plus large. Saisie de questions préjudicielles en interprétation tendant à lui faire apprécier la compatibilité d'une réglementation étatique avec les règles de libre circulation et certains droits fondamentaux, la Cour avait été amenée à préciser les limites de sa compétence. Ce faisant, elle était parvenue, de manière incidente, à imposer de nouvelles contraintes aux Etats membres concernant le respect des droits fondamentaux. Elle avait, en effet, considéré dans un important arrêt de 1991, qu'à partir du moment où une réglementation nationale entrait dans le champ d'application du droit communautaire, la Cour de justice, saisie à titre préjudiciel, devait fournir «tous les éléments d'interprétation nécessaires à l'appréciation, par la juridiction nationale, de la conformité de cette réglementation avec les droits fondamentaux dont la Cour assure le respect» ${ }^{10}$. Tout en confirmant cette prise de position, la Cour avait souligné qu'un Etat membre se prévalant d'objectifs de nature à justifier une réglementation entravant les échanges devait tenir compte des principes généraux du droit et, notamment, des droits fondamentaux ${ }^{11}$.

Les deux lignes de jurisprudence ainsi dégagées par la Cour de justice n'étaient nullement contradictoires. Elles avaient été dégagées dans des contextes différents.

En faisant référence aux expressions de la première ligne de jurisprudence, sortie de son contexte, l'art. 51 de la Charte des droits fondamentaux a eu pour effet de figer les exigences qui avaient été dégagées par la Cour de justice.

Tel que formulé, l'art. 51 de la Charte des droits fondamentaux impose le respect des droits fondamentaux dans la mise en œuvre du droit de l'Union

8 Arrêts du 24 mars 1994, Bostock, C-2/92, EU:C:1994:116, point 16; du 15 février 1996, Duff et a., C-63/93, EU:C:1996:51, point 29 ; du 13 avril 2000, Karlsson et a., C-292/97, EU:C:2000:202, point 37; du 27 juin 2006, Parlement européen cl Conseil UE, C-540/03, EU:C:2006:429, point 105.

9 Arrêt du 13 juillet 2000, Idéal Tourisme, C-36/99, EU:C:2000:405, point 36.

10 Arrêt du 18 juin 1991, ERT, C-260/89, EU:C:1991:254, point 42.

11 Arrêts du 26 juin 1997, Familiapress, C-368/95, EU:C:1997:325, point 24; du 12 juin 2003, Schmidberger, C-112/00, EU:C:2003:333, points 74 et s. 
européenne, ce qui vise notamment une action sur le fondement d'une réglementation de l'Union européenne. Les explications relatives à la Charte des droits fondamentaux, qui doivent être prises en considération par les juridictions européennes et nationales dans l'interprétation de la Charte conformément à l'art. 52, par. 7, de cette dernière, indiquent qu'il découle de la jurisprudence antérieure de la Cour de justice que les Etats membres ne sont tenus de respecter les droits fondamentaux définis dans le cadre de l'Union que lorsqu'ils agissent dans le champ d'application du droit de l'Union. La jurisprudence constructive de la Cour de justice visée dans les explications relatives à la Charte ne devait pas être abandonnée et devait au contraire servir de référence dans différents cas de figure rencontrés.

La notion d'Etats membres est à considérer ici au sens large. Elle désigne toutes les personnes de droit public, ce qui comprend notamment les collectivités territoriales et les établissements publics. Les explications relatives à la Charte indiquent à juste titre que sont tenus au respect de la Charte aussi bien les autorités centrales que les instances régionales ou locales ainsi que les organismes publics. La Charte devrait s'appliquer à toutes les personnes, indépendamment de leur statut de droit public ou de droit privé, qui exercent une mission d'intérêt général et qui sont dotées de prérogatives exorbitantes à l'égard des personnes dont elles régissent l'activité. C'est une telle conception qu'a retenue la Cour de justice au sujet des mesures nationales soumises au respect des libertés fondamentales de circulation garanties par le traité FUE.

Toutes les autorités des Etats membres et des autres personnes morales de droit public doivent être soumises au respect de la Charte. La nature des fonctions exercées, exécutives, législatives ou juridictionnelles, est indifférente aux fins de l'applicabilité de la Charte. La Cour de justice a souligné que le respect de la Charte s'imposait notamment aux juridictions nationales lorsqu'elles mettent en œuvre le droit de l'Union ${ }^{12}$.

L'applicabilité de la Charte des droits fondamentaux aux Etats membres conduira à s'interroger sur la question de savoir si un Etat membre met ou non en ouvre le droit de l'Union européenne, une telle question pouvant prendre plusieurs formes.

Après quelques errements, la jurisprudence de la Cour de justice semble avoir trouvé un cap en vertu de certaines affirmations réitérées à l'occasion de demandes préjudicielles qui soulèvent précisément la question difficile de l'applicabilité de la Charte. Il conviendra de présenter cadre général et critères d'examen des actes étatiques mis en cause (I) avant d'envisager la situation des

12 Arrêt du 5 avril 2016, Aranyosi et Caldararu, C-404/15 et C-659/15, EU:C:2016:198, point 84 . 
actes nationaux d'exécution du droit dérivé (II) et celle des actes nationaux de mise en ouvre des autres sources du droit de l'Union (III).

\section{CADRE GÉNÉRAL ET CRITÈRES D'EXAMEN DES ACTES ÉTATIQUES MIS EN CAUSE}

Ayant à prendre position après que le traité de Lisbonne fut entré vigueur, la Cour de justice fit preuve d'une certaine prudence à l'égard des demandes formulées par les juridictions nationales qui l'interrogeaient sur l'applicabilité de la Charte des droits fondamentaux à des situations où un Etat membre avait prétendument violé le droit de l'Union européenne.

Sans se départir de cette approche prudente et pragmatique, la Cour de justice, en grande chambre, prit une position de principe dans l'affaire Akerberg Fransson au sujet de l'applicabilité de la Charte des droits fondamentaux aux actes des Etats membres de l'Union européenne. Sa position de principe a consisté à retenir une acception large de la notion de mise en œuvre du droit de l'Union ${ }^{13}$.

La Cour de justice a observé que les droits fondamentaux garantis dans l'ordre juridique de l'Union ont "vocation à être appliqués dans toutes les situations régies par le droit de l'Union, mais pas en dehors de telles situations» ${ }^{14}$. Elle a considéré que les droits fondamentaux qui sont garantis par la Charte devaient être respectés «lorsqu'une réglementation nationale entre dans le champ d'application du droit de l'Union ${ }^{15}$. De manière encore plus radicale, la Cour a ajouté que «l'applicabilité du droit de l'Union implique celle des droits fondamentaux garantis par la Charte» ${ }^{16}$.

Ces deux dernières formules, particulièrement significatives, ont été rappelées dans des arrêts ultérieurs ${ }^{17}$, notamment dans des affaires où la réglementation nationale mise en cause ne consistait pas à mettre en œuvre, stricto sensu, le droit de l'Union mais était soumise à des contraintes relevant du

13 Voir notamment à ce sujet Ritleng (2013: 267 et s.) et Epiney (2014: 284 et s.).

14 Arrêt du 26 février 2013, Akerberg Fransson, C-617/10, EU:C:2013:105, point 19. Voir ultérieurement arrêts du 6 octobre 2015, Delvigne, C-650/13, EU:C:2015:648, point 26 ; du 19 novembre 2019, A. K. et a., C-585/18, C-624/18 et C-625/18, EU:C:2019:982, point 78.

15 Arrêt du 26 février 2013, Akerberg Fransson, C-617/10, EU:C:2013:105, point 21.

16 Ibid.

17 Arrêt du 26 septembre 2013, Texdata Software, C-408/11, EU:C:2013:588, point 73; du 30 avril 2014, Pfleger et a., C-390/12, EU:C:2014:281, point 34; du 16 mai 2017, Berlioz Investment Fund, C-682/15, EU:C:2017:373, point 49. 
droit de l'Union européenne et ainsi entrait clairement dans le champ d'application du droit de l'Union.

La notion de mise en ouvre visée à l'art. 51 de la Charte des droits fondamentaux donne ainsi lieu à une appréhension large de la part de la Cour de justice dans la mesure où il suffit a priori que la situation en cause soit régie par le droit de l'Union ou entre dans le champ d'application de ce droit pour que la Charte des droits fondamentaux soit applicable. La formule selon laquelle «les droits fondamentaux garantis dans l'ordre juridique de l'Union ont vocation à être appliqués dans toutes les situations régies par le droit de l'Union, mais pas en dehors de telles situations» ou «de celles-ci» est rappelée avec force dans une jurisprudence constante ${ }^{18}$.

Cette position de principe de la Cour de justice a fait l'objet de vives critiques dans plusieurs Etats membres en ce que la Cour n'avait pas respecté la lettre de l'art. 51 de la Charte des droits fondamentaux et avait ainsi exercé une forme d'hégémonie traduisant un retour à l'activisme dont elle avait fait preuve au cours des années 70 lorsqu'il s'agissait de garantir les droits découlant du marché commun.

Consciente des excès et des abus qui pourraient découler d'une application pure et simple de telles formules, la Cour de justice est venue réitérer des affirmations plus anciennes tout en les systématisant sous la forme de guide ou d'avertissement à l'attention des juridictions nationales, au point que certains observateurs ont pu y déceler une seconde ligne de jurisprudence.

La Cour de justice va ainsi faire usage de formules que l'on pourrait dire «à tiroirs» consistant à introduire plusieurs critères d'appréciation de la condition de «mise en œuvre» établie à l'art. 51 de la Charte.

La Cour de justice a indiqué qu'il y avait lieu de vérifier «parmi d'autres éléments, si la réglementation nationale en cause a pour but de mettre en œuvre une disposition du droit de l'Union, le caractère de cette réglementation et si celle-ci poursuit des objectifs autres que ceux couverts par le droit de l'Union, même s'il existe une réglementation du droit de l'Union spécifique en la matière ou susceptible de l'affecter» ${ }^{19}$. Cette formule sera utilisée avec quelques variantes ${ }^{20}$. L'idée de rattachement au droit de l'Union a pu

18 Arrêt du 27 mars 2014, Torralbo Marcos, C-265/13, EU:C:2014:187, point 29; du 30 juin 2016, Toma et Biroul Excecutorului Judecatoresc Horatiu-Vasile Cruduleci, C-205/15, EU:C:2016:499, point 23; du 19 novembre 2019, A. K. et a., C-585/18, C-624/18 et C-625/18, EU:C:2019:982, point 78.

19 Arrêt du 8 novembre 2012, Ida, C-40/11, EU:C:2012:691, point 79.

20 Arrêts du 8 mai 2013, Ymeraga, C-87/12, EU:C:2013:291, point 41; du 6 mars 2014, Siragusa, C-206/13, EU:C:2014:126, point 25; du 10 juillet 2014, Julian Her- 
ainsi être précisée en ce sens qu'est exigé «un lien de rattachement d'un certain degré, dépassant le voisinage des matières visées ou les incidences indirectes de l'une des matières sur l'autre» ${ }^{21}$.

Suivant l'esprit de cette ligne de jurisprudence, la Cour de justice considère que la seule circonstance qu'une loi étatique est susceptible d'affecter indirectement le fonctionnement d'une organisation commune des marchés ne peut constituer un lien suffisant ${ }^{22}$. Il peut en résulter qu'une réglementation nationale relève d'un domaine dans lequel l'Union dispose de compétences mais que cela ne suffise pas à entraîner l'applicabilité de la Charte compte tenu des facteurs mis en lumière par la Cour de justice ${ }^{23}$.

Ainsi, une réglementation pourtant relative aux droits des travailleurs mais qui poursuit un objectif autre que celui visé par une disposition de l'accordcadre annexé à la directive concernant l'accord-cadre sur le travail à durée déterminée ne peut être considérée comme mettant en œuvre le droit de l'Union européenne ${ }^{24}$. De même, une réglementation nationale qui permet d'accorder des droits à congé excédant la durée minimale prévue par une disposition de la directive sur l'aménagement du temps de travail relève de l'exercice des compétences dites retenues des Etats membres et ne relève pas du champ d'application de la directive et partant de la Charte ${ }^{25}$.

En cas de doute sur l'applicabilité d'un texte de droit européen à une situation donnée, la Cour de justice est encline à considérer qu'elle n'est pas à même de déterminer si la situation du demandeur est régie par le droit de l'Union au sens de l'art. 51 de la Charte ${ }^{26}$.

En présence d'une réglementation nationale qui ne contient aucun élément qui permet de considérer qu'une affaire concerne l'interprétation ou

nandez et a., C-198/13, EU:C:2014:2055, point 37; du 22 janvier 2020, Baldonedo Martin, C-177/18, EU:C:2020:26, point 59.

21 Arrêts du 6 mars 2014, Siragusa, C-206/13, EU:C:2014:126, point 24; du 10 juillet 2014, Julian Hernandez et a., C-198/13, EU:C:2014:2055, point 34; du 17 septembre 2014, Livimaa Lihaveis, C-562/12, EU:C:2014:2229, point 62; du 6 octobre 2016, Paoletti et a., C-218/15, EU:C:2016:748, point 14 ; du 22 janvier 2020, Baldonedo Martin, C-177/18, EU:C:2020:26, point 58.

22 Arrêt du 6 mars 2014, Siragusa, C-206/13, EU:C:2014:126, point 29.

23 Arrêt du 10 juillet 2014, Julian Hernandez et a., C-198/13, EU:C:2014:2055, points 36 et 46.

24 Arrêt du 22 janvier 2020, Baldonedo Martin, C-177/18, EU:C:2020:26, points 61 à 63.

25 Arrêt du 19 novembre 2019, TSN et AKT, C-609/17 et C-610/17, EU:C:2019:981, points 52 et 53 .

26 Arrêt du 17 janvier 2013, Zakaria, C-23/12, EU:C:2013:24, point 39. 
l'application du droit de l'Union autre que celles des dispositions de la Charte, la Cour de justice se déclarera incompétente pour répondre à la demande de renvoi préjudiciel ${ }^{27}$.

La jurisprudence de la Cour de justice invite à s'interroger sur les différentes sources du droit de l'Union européenne qui donnent lieu à une telle mise en œuvre de la part des Etats membres.

Il est des cas où la condition ainsi posée ne soulève guère de difficultés au motif que la réglementation nationale est une mesure d'application d'un texte de droit dérivé de l'Union européenne. Il en est d'autres où ladite condition suscite de nombreuses interrogations en présence d'autres sources que celles de droit dérivé de l'Union à mettre en œuvre.

\section{EXAMEN DES ACTES ÉTATIQUES DE MISE EN OEUVRE DU DROIT DÉRIVÉ DE L'UNION EUROPÉENNE}

Le droit dérivé de l'Union européenne ayant force contraignante est constitué principalement de règlements, de directives et de décisions adoptées par les institutions de l'Union. De tels actes sont soumis au respect de la Charte des droits fondamentaux en vertu de son art. 51, par. $1^{\text {er }}$. La plupart de ces actes peuvent, en fonction de leurs finalités et de leur portée, nécessiter des actes étatiques de mise en œuvre qui, à leur tour, devraient être soumis au respect des dispositions de la Charte des droits fondamentaux dans la mesure où ils constituent le prolongement des actes des institutions de l'Union européenne.

Même s'ils sont d'applicabilité directe en vertu de l'art. 288, alinéa 2, TFUE, les règlements européens nécessitent souvent des mesures d'application de la part des Etats membres. Avant l'adoption de la Charte des droits fondamentaux, la jurisprudence de la Cour de justice avait souligné l'exigence de respect des droits fondamentaux imposée aux Etats membres lorsqu'ils mettaient en œuvre une réglementation européenne dans le cadre d'une politique ou d'une action commune de l'Union européenne ${ }^{28}$. Cette

27 Ordonnacces du 8 mai 2014, Pelckmans Turnhout, C-483/12, EU:C:2014:304, points 22 à 27; du 25 février 2016, Aiudapds, C-520/15, EU:C:2016:124, points 20 et 21; du 26 octobre 2017, Caixa Economica Montepio Geral, C-333/17, EU:C:2017: 810 , points 16 à 19 .

28 Arrêts du 24 mars 1994, Bostock, C-2/92, EU:C:1996:51, point 16; du 13 avril 2000, Karlsson et a., C-292/97, EU:C:2000:202, point 17. 
jurisprudence a logiquement été reprise à l'identique au sujet des droits consacrés dans la Charte des droits fondamentaux ${ }^{29}$.

Il aurait été possible d'introduire des nuances tenant compte de la marge d'appréciation laissée aux Etats membres en vertu de cette réglementation européenne mais l'établissement d'un seuil aurait été très difficile à établir et aurait pu nuire à la sécurité juridique. Aussi la Cour de justice a-t-elle fort logiquement considéré qu'il n'y avait pas lieu de distinguer selon que l'Etat membre en cause avait ou non une marge d'appréciation au regard du règlement de l'Union européenne qu'il est en charge d'appliquer ${ }^{30}$. Ainsi, la décision d'un État membre de transférer un demandeur, en application du règlement Dublin III concernant l'examen des demandes de protection internationale, vers l'État membre qui est en principe responsable de l'examen de la demande de protection internationale constitue un élément du système européen commun d'asile et, par conséquent, met en œuvre le droit de l'Union ${ }^{31}$ sans qu'il y ait lieu de s'interroger sur l'étendue de la marge d'appréciation dont il disposait.

De même, la Cour de justice a précisé que le pouvoir de dérogation reconnu à un Etat membre en vertu d'un règlement européen, tel que le règlement Dublin III, relève logiquement de la mise en œuvre du droit de l'Union ${ }^{32}$. La question pourrait alors se poser de savoir si cette exigence vaut également lorsqu'un Etat membre exerce un pouvoir de dérogation non expressément reconnu par un règlement de l'Union. Selon nous, il devrait en aller de même, faute de quoi les Etats membres auraient tout intérêt à exercer leurs prérogatives au-delà de celles qui sont expressément reconnues par les textes de droit dérivé de l'Union pour échapper aux contraintes de la Charte. L'essentiel est, selon nous, que l'Etats membres agissent dans le champ d'application d'un règlement de l'Union en vue de s'y conformer et de lui donner effet, conformément à la ligne de jurisprudence Akerberg Fransson ${ }^{33}$.

La condition de mise en œuvre du droit de l'Union n'est pas satisfaite, selon la Cour de justice, lorsqu'une réglementation européenne adoptée dans le cadre d'une politique, telle que la politique agricole commune, ne contient ni ne renvoie à des dispositions sur la réparation des dommages,

29 Arrêt du 17 septembre 2014, Livimaa Lihaveis, C-562/12, EU:C:2014:2229, point 65.

30 Arrêt du 21 décembre 2011, N. S. et a., C-411/10 à C-493/10, EU:C:2011:865, point 68 .

31 Arrêt du, 19 mars 2019, Jawo, C-163/17, EU:C:2019:218, point 77.

32 Arrêt du 16 février 2017, C.K. et a., C-578/16 PPU, EU:C:2017:127, point 53.

33 Voir supra note 6. 
ces dispositions de droit national n'étant pas régies par le droit de l'Union européenne $e^{34}$. On aurait pu soutenir qu'elles découlaient ou qu'elles étaient «impliquées» par la réglementation européenne, en observant que l'on ne peut contraindre les opérateurs économiques sans envisager leur responsabilité qui découle des violations de cette réglementation. Telle n’a pas été la conception de la Cour de justice qui a fait preuve de retenue.

Dans la mesure où elles nécessitent des mesures de transposition en vertu de l'art. 288, alinéa 3, TFUE, les directives adoptées par les institutions de l'Union européenne donneront lieu à des mesures de «mise en œuvre» par les Etats membres. La Cour de justice avait déjà soumis les mesures de transposition d'une directive au respect des droits fondamentaux avant l'adoption de la Charte des droits fondamentaux ${ }^{35}$. Elle a confirmé qu'une réglementation nationale de transposition d'une directive constitue une opération de mise en œuvre du droit de l'Union ${ }^{36}$. De même, les mesures prises par les autorités judiciaires d'émission ou d'exécution appliquant les dispositions nationales prises en application de la décision-cadre relative au mandat d'arrêt européen constituent des mesures de mise en œuvre du droit de l'Union et doivent ainsi respecter tant les principes généraux du droit ${ }^{37}$ que les dispositions de la Charte $^{38}$.

La Cour de justice a interprétélargement la notion de mise en œuvre d'une directive en ne se limitant pas aux seules mesures de transposition stricto sensu qu'elle requiert précisément. Des sanctions fiscales et des poursuites pénales pour fraude fiscale, non directement fixées par une directive européenne, constituent la mise en ouvre d'un dispositif fiscal, celui de la TVA, dont les recettes alimentent le budget de l'Union, et, de manière indirecte, l'obligation établie par l'art. 325 TFUE à l'endroit des Etats membres de lutter contre les activités illicites portant atteinte aux intérêts financiers de l'Union ${ }^{39}$. De même, une sanction pécuniaire qui vise à garantir le respect d'une directive, alors qu'elle n'est pas expressément prévue par cette dernière, constitue la

34 Arrêt du 22 mai 2014, Ersekcsanadi Mezogazdasagi, C-56/13, EU:C:2014:352, point 57.

Arrêts du 6 novembre 2018, Bauer et Brosson, C-569/16 et C-570/16, EU:C:2018:871, point 53; du 6 novembre 2018, Max-Planck-Gesellschaft, C-684/16, EU:C:2018:874, point 50; du 8 mai 2019, Leitner, C-396/17, EU:C:2019:375, point 58. Arrêt du 5 avril 2016, Aranyosi et Caldararu, C-404/15 et C-659/15, EU:C:2016:198, point 84 .

39 Arrêts du 26 février 2013, Akerberg Fransson, C-617/10, EU:C:2013:105, point 27; du 5 avril 2017, Orsi et Baldetti, C-217/15 et C-350/15, EU:C:2017:264, point 16; du 20 mars 2018, Menci, C-524/15, EU:C:2018:197, point 21. 
mise en œuvre de la directive ${ }^{40}$. Des poursuites pénales qui visent à réprimer l'aide à l'immigration illégale réglementée par plusieurs actes de droit dérivé de l'Union constituent une mise en ouvre de ces actes ${ }^{41}$. Il est à cet égard indifférent que la disposition nationale qui sert de fondement à la sanction n'ait pas été adoptée en vue de transposer la directive en cause ${ }^{42}$.

Il reste qu'en dépit de telles affirmations relatives aux règlements, aux directives et aux décisions-cadre, la condition de mise en ouvre de droit de l'Union pourra présenter des difficultés d'interprétation dans des situations où il convient de s'attacher non seulement aux rapports entre le texte de droit dérivé de l'Union et les textes nationaux mais à la situation en cause dans le cadre du litige, ce qui pourra notamment être le cas dans des affaires pénales ou interindividuelles.

La mise en lumière des circonstances de l'espèce pourra être privilégiée pour ne pas rendre applicable la Charte à une législation qui pouvait pourtant consister objectivement en une mise en ouvre du droit de l'Union.

Ainsi, dans l'affaire $I d a$, concernant un ressortissant japonais qui vivait séparé de son épouse de nationalité allemande et qui s'était vu refuser un titre de séjour de membre de la famille d'un citoyen de l'Union en Allemagne en raison de la rupture de la communauté de vie, le ressortissant n'était a priori plus en droit de bénéficier directement ou indirectement du statut de citoyen de l'Union régi par la directive, ce qui a sans doute été décisif aux yeux de la Cour de justice pour lui dénier la possibilité de se prévaloir de la Charte des droits fondamentaux ${ }^{43}$. La Cour de justice a considéré que bien que le texte visé relatif à la délivrance d'un tel titre ait visé à mettre en œuvre le droit de l'Union, il y avait lieu de prendre en considération la situation du demandeur qui n'était pas régie par le droit de l'Union européenne, dans la mesure où il ne remplissait pas les conditions prévues pour l'octroi d'une carte de séjour au titre de la directive ${ }^{44}$. Ainsi, dans la mesure où un sujet de droit ne remplit pas les conditions définies par un texte de droit de l'Union européenne pour en obtenir le bénéfice, il ne peut pas faire valoir que le texte national qui lui est

40 Arrêts du 26 septembre 2013, Texdata Software, C-408/11, EU:C:2013:588, points 74 et 75; du 16 mai 2017, Berlioz Investment Fund, C-682/15, EU:C:2017:373, point 39.

41 Arrêt du 6 octobre 2016, Paoletti et a., C-218/15, EU:C:2016:748, point 18.

42 Arrêts du 26 février 2013, Akerberg Fransson, C-617/10, EU:C:2013:105, point 28; du 6 octobre 2016, Paoletti et a., C-218/15, point 18; du 16 mai 2017, Berlioz Investment Fund, C-682/15, EU:C:2017:373, point 40.

43 Arrêt du 8 novembre 2012, Ida, C-40/11, EU:C:2012:691, point 80.

44 Arrêt du 8 novembre 2012, Ida, C-40/11, EU:C:2012:691, point 80. 
opposé est un texte de mise en œuvre de ce texte de droit de l'Union, même si objectivement tel est bien le cas.

Dans d'autres cas de figure, il apparaissait clairement que les sujets de droit qui s'étaient prévalu des dispositions de la Charte devant la juridiction nationale à l'origine des questions préjudicielles appartenaient au champ des bénéficiaires de certains droits ouverts par des dispositions du droit de l'Union européenne. Ainsi, dans l'affaire Akerberg Fransson, il apparaissait que l'entreprise en cause était en droit de bénéficier du statut d'assujetti à la TVA en vue de contester les poursuites pénales au regard de la Charte des droits fondamentaux $^{45}$.

Ce type d'élément devrait être mis en lumière plus nettement par la Cour de justice de manière à ce que l'on comprenne que seuls ceux qui sont précisément en droit de se prévaloir d'une réglementation du droit de l'Union européenne peuvent invoquer la Charte des droits fondamentaux lorsqu'ils contestent des actes étatiques de mise en œuvre de cette réglementation. On pourrait certes observer que la Cour de justice n'est pas en charge de statuer sur ces questions lorsqu'elle est saisie d'une demande de renvoi préjudiciel mais on pourrait alors objecter que sa connaissance des éléments factuels et réglementaires nationaux acquise en vertu de la décision de renvoi, conformément aux prescriptions du règlement de procédure de la Cour de justice, et des pièces du dossier qui ont pu être transmises lui permettent de porter des appréciations de manière incidente au regard de ces éléments ${ }^{46}$.

Les difficultés pourront se manifester avec plus d'acuité lorsque la mise en œuvre ne se rapporte pas au droit dérivé mais à d'autres sources de droit de l'Union européenne.

\section{EXAMEN DES ACTES ÉTATIQUES DE MISE EN OEUVRE DES AUTRES SOURCES DE DROIT DE L'UNION EUROPÉENNE}

La contestation, au regard de la Charte des droits fondamentaux de l'Union européenne, des actes étatiques qui ne sont pas en relation directe avec la réglementation de l'Union européenne a pu paraitre inappropriée ou abusive aux yeux de plusieurs observateurs. La condition de mise en œuvre du droit de l'Union européenne établie par l'art. 51 de la Charte a en effet été pensée principalement en relation avec la réglementation de l'Union

45 Voir supra I.

46 Voir notamment sur ces questions Picod et Rideau (2017: 195-208). 
européenne laquelle est pleinement soumise au respect des dispositions de la Charte.

Pour autant, il y a lieu de s'interroger sur l'applicabilité de la Charte à des actes étatiques qui ne mettent pas en œuvre du droit dérivé mais sont en relation directe avec d'autres sources du droit de l'Union européenne. A ce titre, il conviendrait de prendre en considération la finalité des normes qu'elles véhiculent.

Les partisans d'une applicabilité restreinte de la Charte des droits fondamentaux aux Etats membres pourraient faire observer qu'elle ne leur est pas applicable lorsqu'ils mettent en œuvre une source de droit de l'Union européenne, telle que les traités constitutifs de l'Union européenne, qui n'est elle-même pas soumise au respect de la Charte. Une telle thèse ne nous paraît toutefois pas conforme à l'esprit de la jurisprudence de la Cour de justice développée avant l'adoption de la Charte et confirmée depuis lors au sujet de l'interprétation de son art. 51, par. $1^{\mathrm{er}}$.

Lorsque des sources contraignantes de droit de l'Union européenne produisent des effets comparables à ceux du droit dérivé de l'Union, tout particulièrement dans l'encadrement de l'activité économique et sociale, il n'y a pas lieu selon nous d'adopter une solution différente de celle exposée précédemment ${ }^{47}$.

Ainsi, s'agissant de la décision adoptée par les Etats membres en 1976 relative à l'élection des représentants des peuples des Etats membres au suffrage universel direct au Parlement européen, laquelle s'intègre au droit primaire du droit de l'Union européenne, une législation étatique qui exclut des citoyens du nombre de ceux qui ont le droit de vote à ces élections met en œuvre l'acte de 1976, lu en rapport avec l'art. 14 TUE qui impose d'assurer l'élection au suffrage universel direct libre et secret ${ }^{48}$.

De même, au sujet d'un protocole d'accord entre l'Union européenne et un Etat membre ayant ultérieurement adhéré à l'Union européenne, qui fixe des objectifs précis à mettre en œuvre par les partenaires, la Cour de justice constate que ce protocole donne lieu à une loi de mise en œuvre qui est dès lors soumise au respect des dispositions de la Charte ${ }^{49}$.

A l'avenir, la question de l'applicabilité de la Charte des droits fondamentaux devrait se poser au sujet d'accords internationaux qui lient l'Union européenne et des Etats tiers, notamment d'accord mixtes qui sont conclus parallèlement par l'Union et les Etats membres avec des Etats tiers, lesquels

\footnotetext{
47 Voir supra II.

48 Arrêt du 6 octobre 2015, Delvigne, C-650/13, EU:C:2015:648, point 33.

49 Arrêt du 13 juin 2017, Florescu et a., C-258/14, EU:C:2017:448, point 53.
} 
donnent lieu de la part des Etats membres à des mesures d'application se rapportant, par exemple, au séjour des ressortissants, à leur protection sociale ou à la fiscalité de leurs biens. Dès lors que de telles mesures mettent en ouvre des dispositions d'un accord qui relèvent pleinement de la compétence de l'Union européenne, elles devraient être soumises, au même titre que les dispositions de ces accords, au respect de la Charte. La question pourrait toutefois se poser de savoir si les questions traitées par les Etats membres en application d'un accord mixte, au sujet des ressortissants des Etats tiers, se rapportent à des domaines de compétence de l'Union ou à celle des Etats membres pour envisager l'applicabilité de la Charte des droits fondamentaux.

Lorsque le seul élément du droit de l'Union européenne invocable se situe dans les traités constitutifs de l'Union, il y a lieu de se demander s'il suffit en vue de considérer que la disposition du traité est le fondement ou le support d'un acte de «mise en œuvre» par les Etats membres.

Il y aurait lieu, selon nous, de prendre en considération la finalité et la portée de la disposition considérée du traité constitutif de l'Union européenne.

Lorsqu'il s'agit d'un art. du traité qui se limite à permettre l'adoption d'une réglementation européenne, dans un domaine de compétence partagée ou d'appui ou de soutien, et que cette réglementation n'a pas encore été adoptée, il nous paraît inapproprié de considérer que le droit national applicable en la matière constitue la mise en ouvre de l'article du traité au seul motif qu'il existe une identité de matière.

Lorsqu'il s'agit d'un article du traité qui prescrit un comportement étatique ou qui l'interdit, il nous paraît au contraire logique de considérer que l'action étatique et, plus largement, celle de la puissance publique constitueront une mise en œuvre de cet article du traité. Ce devrait être le cas des articles du traité FUE qui contraignent les Etats membres dans le cadre des libertés fondamentales de circulation, des aides d'Etat, de l'aménagement des monopoles à caractère commercial, des impositions fiscales, des droits de la citoyenneté de l'Union, de la non-discrimination et plus largement du respect des droits fondamentaux. Le champ d'invocabilité de la Charte des droits fondamentaux pourrait ainsi être considérablement élargi.

La confrontation d'une réglementation à l'une des libertés fondamentales de circulation garanties par le traité FUE, le cas échéant avec la revendication d'une justification prévue par le traité ou reconnue par la jurisprudence, relève du champ d'application du droit de l'Union et, par conséquent, du 
champ de la Charte des droits fondamentaux ${ }^{50}$, suivant la ligne de raisonnement dégagée dans l'arrêt Akerberg Fransson ${ }^{51}$.

Dans de tels cas de figure, la réglementation européenne fait défaut. Les textes de droit de l'Union applicables sont des dispositions des traités constitutifs qui prohibent des comportements restrictifs. La Cour de justice prend appui également sur sa jurisprudence dégagée antérieurement à la Charte ${ }^{52}$. Elle considère qu'une réglementation nationale ne peut bénéficier d'une justification que si elle est conforme aux droits fondamentaux garantis dans l'ordre juridique de l'Union européenne ${ }^{53}$. La Cour pend soin de préciser que le recours, par un Etat membre, à des exceptions (le terme exact étant celui de dérogations) prévues par le droit de l'Union européenne pour justifier une entrave à une liberté fondamentale garantie par le traité constitutif est à considérer comme «mettant en ouvre le droit de l'Union" ${ }^{54}$.

Il arrive toutefois que le lien avec les libertés de circulation ne soit pas suffisamment établi, ce qui prive les justiciables de la possibilité d'invoquer la Charte. Dans le cadre d'un renvoi préjudiciel, la Cour considère que la demande doit faire ressortir des éléments concrets, notamment des indices certains, permettant d'établir, de manière positive, l'existence d'un intérêt émanant de ressortissants d'autres États membres à faire usage de ces libertés fondamentales dans la situation en cause ${ }^{55}$.

De même, si les effets sur les libertés de circulation en cause sont trop aléatoires et indirects pour les appliquer à la réglementation en cause, cette dernière ne pourra pas être confrontée à la Charte ${ }^{56}$.

Dans une situation purement interne, concernant une amende infligée par un Etat membre à l'un de ses ressortissants qui se déplaçait sur son territoire, la Cour de justice a considéré à juste titre que la réglementation nationale en cause ne constituait pas "une mesure de mise en œuvre du droit de l'Union"

50 Arrêt du 30 avril 2014, Pfleger et a., C-390/12, EU:C:2014:281, point 36.

51 Voir supra I.

52 Arrêts du 18 juin 1991, ERT, C-260/89, EU:C:1991:254, point 42; du 26 juin 1997, Familiapress, C-368/95, EU:C:1997:325, point 24; du 12 juin 2003, Schmidberger, C-112/00, EU:C:2003:333, points74 et s. Voir déjà à ce sujet Kaddous (2007: 397 et s.), Kombas (2006: 433 et s.), Shuibhne (2009: 230 et s.) et Skouris (2006: 497 et s.)

53 Arrêts du 30 avril 2014, Pfleger et a., C-390/12, EU:C:2014:281, point 35; du 21 mai 2019, Commission c/ Hongrie, C-235/17, EU:C:2019:432, point 64.

54 Arrêt du 21 mai 2019, Commission c/ Hongrie, C-235/17, EU:C:2019:432, point 65.

55 Arrêts du 20 septembre 2018, Fremoluc, C-343/17, EU:C:2018:754, points 28 et 29;

CJUE, 15 novembre 2018, Nova Kreditna Banka Maribor, C-215/17, EU:C:2018: 901 , points 44 et 45 .

56 Arrêt du 8 mai 2014, Pelckmans Turnhout, C-483/12, EU:C:2014:304, point 25. 
et ne présentait pas "d'autres éléments de rattachement à ce droit» ${ }^{57}$, une telle formule étant toutefois susceptible d'introduire le doute.

Au-delà même de la confrontation d'une réglementation étatique à un art. contraignant du traité constitutif, tel que l'art. 110 du traité FUE qui interdit les impositions fiscales discriminatoires ou protectrices, l'action en répétition de l'indu des sommes acquittées en application de la réglementation nationale en violation de cet art. ou en exécution d'une décision de justice y relative sera soumise au respect de la Charte des droits fondamentaux ${ }^{58}$. Ici encore, on pourrait considérer que l'action en répétition de l'indu ou l'action en exécution est le prolongement logique de la violation d'une disposition du traité constitutif qui interdit certaines impositions fiscales discriminatoires ou protectrices de marchandises.

On pourra alors s'interroger sur la question de savoir si une action introduite par un opérateur sur le fondement du droit national qui n'est pas dans le prolongement logique de la disposition du traité constitutif qui sert de référence peut être assimilée à une mise en ouvre de ce dernier. La réponse à une telle question devrait, selon nous, dépendre de l'intensité du lien de rattachement de l'acte étatique mis en cause avec les dispositions du traité constitutif qui servent de fondement à l'action mise en œuvre.

S’il apparaît que la disposition du traité FUE invoquée par un justiciable pour contester une mesure nationale n'est pas applicable à la situation en cause, au motif par exemple que cette dernière est purement nationale ou que l'activité confrontée au droit de la concurrence ou à la libre prestation de services est dénuée de caractère économique, la condition dite de mise en ouvre du droit de l'Union ne saurait être satisfaite et la Charte des droits fondamentaux ne serait pas applicable.

Il reste à se demander si les dispositions énoncées dans la Charte des droits fondamentaux de l'Union européenne peuvent elles-mêmes constituer des sources de référence à la mise en œuvre du droit de l'Union par les Etats membres. Dans la mesure où les dispositions de la Charte énoncent non seulement des droits mais également des principes lesquels, en vertu de son art. 52, par. 5, "peuvent être mises en œuvre par des actes législatifs et exécutifs par les institutions, organes et organismes de l'Union, et par des Etats membres lorsqu'ils mettent en ouvre le droit de l'Union", il y aura de s'interroger sur le sens à donner à cette dernière expression. On pourrait considérer que cela consiste à imposer aux Etats membres de mettre en œuvre

57 Arrêt du 7 juin 2012, Vinkov, C-27/11, EU:C:2012:326, point 59.

58 Arrêt du 30 juin 2016, Toma et Biroul Excecutorului Judecatoresc Horatiu-Vasile Cruduleci, C-205/15, EU:C:2016:499, point 28. 
une disposition de droit dérivé ou une disposition contraignante du traité constitutif en plus de mettre en œuvre un principe énoncé dans la Charte pour que celle-ci soit applicable, ce qui serait très exigeant. Suivant une interprétation plus souple, on pourrait observer que la précision apportée à l'art. 52, par. 5, n'est qu'une redite par rapport à la prescription de l'art. 51, par. $1^{\text {er }}$, de la Charte et qu'il suffit dès lors qu'un principe clairement identifié dans la Charte soit mis en ouvre par un Etat membre au moyen d'un acte explicite pour que ce dernier soit soumis au respect du principe ainsi mis en ouvre et soit tenu plus largement au respect d'autres dispositions de la Charte.

Comme la Cour de justice l'a justement souligné, l'objectif de la protection des droits fondamentaux dans le droit de l'Union est de veiller à ce que ces droits ne soient pas violés dans les domaines d'activités de l'Union, que ce soit en raison de l'action de l'Union ou en raison de la mise en œuvre du droit de l'Union par les Etats membres ${ }^{59}$. La nature du domaine d'activité de l'Union européenne et la forme prise pour son action ne nous paraissent pas décisives, dès lors que les Etats membres agissent directement dans le champ d'action de l'Union. La Cour de justice ne devrait pas se montrer trop exigeante au sujet du lien requis entre l'action de l'Union européenne et l'action étatique, faute de quoi les exigences de la Charte ne pourraient plus être imposées aux Etats membres dans un grand nombre de situations et une importante insécurité juridique pourrait en résulter.

\section{Bibliographie}

Besselink, F.M.L. (2001). The Member States, the national constitutions and the scope of the Charter. Maastricht Journal of European and Comparative Law, 1, 68-80. Disponible en: https://doi.org/10.1177/1023263X0100800105.

Borowsky, M. (2014). Artikel 51. En J. Meyer (éds.). Charta der Grundrechte der Europäischen Union (743-777). Baden-Baden: Nomos.

Coppel, J., O'Neill, A. (1992). The European Court of Justice: Taking Rights Seriously?. Common Market Law Review, 29 (4), 669-692. Disponible en: https://doi.org/10.1111/j.1748-121X.1992.tb00467.x.

Epiney, A. (2014). Le champ d'application de la Charte des droits fondamentaux: l'arrêt Fransson et ses implications. Cahiers de droit européen, 50 (2), 283-303.

Kaddous, C. (2007). Les droits de l'homme et les libertés de circulation en droit communautaire. Revue suisse de droit international et européen 3, 397.

Kombas, C. (2006). Fundamental Rights and Fundamental Freedom: A symbiosis on the Basics of subsidiarity. European Public Law, 12, 433.

59 Arrêt du 6 mars 2014, Siragusa, C-206/13, EU:C:2014:126, point 31. 
Lenaerts, K. (2017). Le champ d'application de la Charte des droits fondamentaux de l'Union européenne. En Liber amicorum Rusen Ergec (pp. 217-228). Luxembourg: Pasicrisie luxembourgeoise.

Ovadek, M. (2017). Le champ d'application de la Charte des droits fondamentaux de l'Union européenne et les États membres. Journal des Tribunaux-Droit Européen, 10, 386-390.

Picod, F. (2018). La mise en œuvre du droit de l'Union européenne. Condition énigmatique d'applicabilité de la Charte des droits fondamentaux. En Mélanges en l'honneur du Professeur Frédéric Sudre (pp. 559-567) Paris: Lexis Nexis.

- (2019). Article 5. En F. Picod, C. Rizcallah, S. Van Drooghenbroeck (dirs.), Charte des droits fondamentaux de l'Union européenne. Commentaire article par article (pp. 1223-1248). Bruxelles: Bruylant.

Picod, F. et Rideau, J. (2017). Renvoi préjudiciel. En Répertoire de droit européen. Paris: Dalloz.

Rideau, J. (dir.) (2009). Les droits fondamentaux dans l'Union européenne. Dans le sillage de la Constitution européenne. Bruxelles: Bruylant.

Ritleng, D. (2013). De l'articulation des systèmes de protection des droits fondamentaux dans l'Union. Les enseignements des arrêts Akerberg Fransson et Melloni. Revue trimestrielle de droit européen, 49 (2), 267-292.

Safjan, M. T., Düsterhaus, D., Guerin, A. (2016). La Charte des droits fondamentaux de l'Union européenne et les ordres juridiques nationaux, de la mise en œuvre à la mise en balance. Revue trimestrielle de droit européen. 2, 219-247.

Shuibhne, N.N. (2009). Margins of appreciation: National values, fundamental rights and EC free movement law. European Law Review, 34 (2), 230-256.

Skouris, V. (2006). L'interaction entre les libertés fondamentales et les libertés publiques en droit communautaire. En Mélanges en l'honneur de Philippe Léger (pp 497-506). Paris: A. Pédone.

Tizzano, A. (2014). L'application de la Charte des droits fondamentaux dans les États membres à la lumière de son article 51, paragraphe 1 . Il Diritto dell'Unione Europea 3, 429-437.

Ward, A. (2014). Article 51. En S. Peers, T. Harvey, J. Kenner, A. Ward, A. (Eds.), The EU Charter of Fundamental Rights. A Commentary (pp. 1413-1454). München: Beck.

Weiler, J. H. H.; Lockhart, N. J. S. (1995). Taking Rights Seriously: The European Court and its Fundamental Rights Jurisprudence. Common Market Law Review, 32, 51 (part I) et 579 (part II). 